\title{
SOBRE LOS ROBLEDALES DEL LLANO DE LA SELVA (GERONA)
}

\author{
Luis VILAR \& Xavier VIÑAS
}

RESUMEN: El Llano de la Selva (Gerona) es una depresión que presenta condiciones climáticas y geológicas especiales que favorecen al bosque caducifolio frente al perennifolio de las Sierras vecinas. Se trata de robledales de Quercus pubescens de los cuales se describe una nueva subasociación.

Palabras clave: Fitosociología, Bosques, Robledales de Q. gr. pubescens, La Selva (Cataluña), Quercion ilicis, Quercion robori-petraeae..

SUMMARY: Two oak groves from Selva country (NE part of Iberian Peninsula) has been studied. The central part of this country (Selva Depression) have special geological and climatical characteristics which favour decidous species instead evergreen ones, which are common in the neighbouring mountains.

Key words: Phytosociology, Forests, Oak groves of Q.gr. pubescens, Quercion ilicis, Quercion robori-petraeae.

\section{N T R O D U C C I O N}

Algunas llanuras aluviales de la cuenca mediterránea, mayoritariamente zonas de cultivo en la actualidad, debían de asentar frondosos y espesos bosques en los cuales las especies caducifolias tendrían un papel principal respecto a las perennifolias. El hecho es conocido de diversos puntos: el Languedoc francés (Kielhauser, 1939 y Braun-Blanquet et al., 1952), el Mediterráneo Oriental (Quèzel, 1985)... En algunos puntos de la provincia de Gerona los bosques de llano aluvial son aún importantes en el paisaje y su estudio, a la vez que contribuye a la caracterización de la vegetación potencial del territorio, aporta nuevos datos para establecer el papel del roble y otras especies caducifolias en suelos sedimentarios mediterráneos.

Situación. La Depresión de la Selva se sitúa en el extremo septentrional de las Sierras Costeras Catalanas, desde la ciudad de Girona hasta el río Tordera, en el límite con la provincia de Barcelona (sector Vallesano-Empordanés). Geomorfológicamente, constituye un bloque deprimido de unos $200 \mathrm{Km}^{2}$ de superficie, entre 70 y 200 metros de altitud, rodeado de otros bloques elevados, todos ellos de naturaleza granítica y 
esquistosa: el macizo del Montseny-Guilleries (Cordillera Prelitoral) y Les Gavarres y Sierra de Marina (Cordillera Litoral).

El sustrato. El Llano de la Selva constituye una antigua zona endorreica recubierta por sedimentos pliocénicos y cuaternarios de naturaleza arcósica y arcillosa, resultantes de la erosión de las Sierras vecinas. Estos sedimentos configuran un relieve ondulado de pendiente suave y suelos profundos.

También existen numerosos afloramientos de materiales volcánicos como resultado de las fallas que fracturaron la zona y delimitaron la depresión durante el Plioceno, así como facies de granodiorita y granito biotítico a menudo recubiertas por los sedimientos.

El clima. La comarca de la Selva presenta un clima de tipo mediterráneo xerotérico de tendencia húmeda, según Bolòs et Vigo (1984) o clima mesomediterráneo según la clasificación bioclimática de Rivas Martínez (1987).

La Depresión de la Selva, sin embargo, presenta una característica particular que no se refleja en los diagramas climáticos: la presencia de una acusada inversión térmica invernal, la cual provoca abundantes nieblas y temperaturas más bajas que las registradas en las sierras vecinas.

Según hemos podido calcular (Vilar et al., en prensa) puede llegar a registrarse una diferencia de temperatura de hasta $9^{\circ} \mathrm{C}$ entre las partes más bajas del Llano y las zonas más elevadas circundantes. Estas temperaturas menores y los factores edáficos, especialmente la disponibilidad de nutrientes, cuyas características habrá que estudiar más detenidamente algun día, favorecerían, desde nuestro punto de vista, las especies caducifolias frente a las de hoja perenne.

La vegetación. Varios tipos de vegetación forman en el llano un variado mosaico atendiendo a las diferencias microclimáticas y a los diversos tipos de sustrato. Puede verse esquematizada su disposición en la fig. 1. La vegetación climatófila comprende el robledal acidófilo del Llano (1), los bosques mixtos de encinas y robles (2), el encinar (23), el alcornocal (4), y el robledal acidófilo (5), mientras que la edafófila comprende la vegetación de marjal (6) y el bosque de ribera (7).

Excepto las dos primeras formaciones, las demás tienen un papel marginal en el llano y ya han sido estudiadas por varios autores (Zeller, 1959 y Bolòs, 1959), por lo que nos centraremos aquí, únicamente, en los robledales del llano (bien sean puros o mezclados con encinas), en los que el roble tiene un papel destacado.

\section{R E S U L T A D OS .}

\section{Carici-Quercetum canariensis O. Bolòs 1954 holcetosum nova.}

Robledal acidófilo del llano (Inventarios n⿳ำ 14-18 de la tabla adjunta). Esta comunidad, que describimos como subasociación holcetosum por la relativa abundancia de Holcus mollis, ocupa las partes más bajas del llano, de suelo cuaternario arcilloso y húmedo gran parte del año aunque nunca inundado.

El estrato arbóreo presenta un aspecto denso (recubrimientos entre 60 y 90\%) y está dominado por Quercus pubescens (inventario tipo $\mathrm{n}^{\circ}$ 16). Pinus pinea, introducido pero sin regeneración natural, está casi siempre presente y Quercus ilex es, en cambio, escaso. En el estrato arbustivo dominan Erica scoparia y E. arborea aunque 


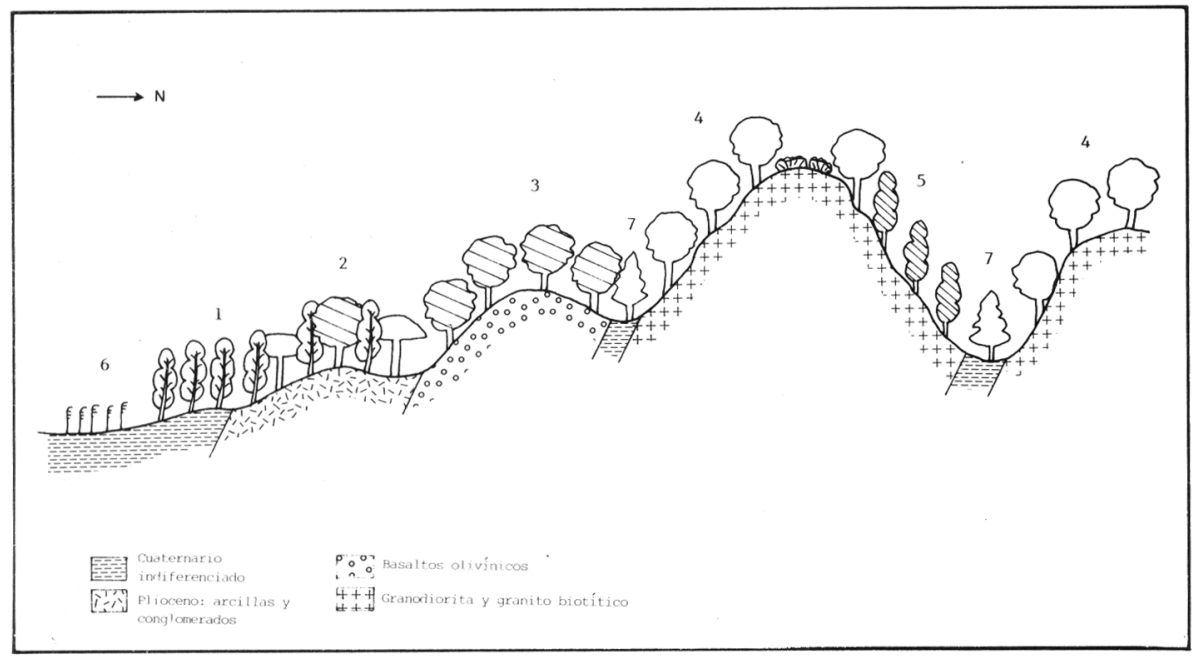

Fig. 1.- Transecto esquemático de la vegetación del Llano de la Selva: 1. Robledal acidófilo del llano (Carici-Quercetum canariensis holcetosum), 2. Bosque mixto de encinas y robles (Quercetum ilicis galloprovinciale quercetosum pubescentis), 3. Encinar (Quercetum ilicis galloprovinciale pistacietosum), 4. Alcornocal (Quercetum ilicis galloprovinciale suberetosum), 5. Robledal acidófilo (Carici-Quercetum canariensis), 6. Vegetación de marjal (mosaico de Typho-Schoenoplectetum glauci, Cypero Caricetum otrubae y GaudinioArrhenatheretum) y 7 . Aliseda (Lamio-Alnetum glutinosae).

las especies de Querco-Fagetea tienen una buena representación (Juniperus communis ssp. communis, Corylus avellana, Prunus spinosa y Crataegus monogyna). El estrato herbáceo está dominado por especies del Quercion robori-petraeae (Serratula tinctoria, Hieracium gr. sabaudum, Lonicera peryclimenum y Holcus mollis) y contiene numerosas plantas de Querco-Fagetea (Euphorbia amygdaloides, Brachypodium sylvaticum, Stachys officinalis, etc.) así como un buen número de especies mediterráneas (Rubia peregrina, Rosa sempervirens y Asparagus acutifolius).

Inscribimos esta asociación dentro del Quercion robori-petraeae por la importancia que tienen en ella las especies de dicha alianza. Y, a pesar de la ausencia de $Q$. canariensis, en el Carici-Quercetum canariensis por la afinidad florística y biogeográfica con la asociación descrita por O. Bolòs (1954) sobre roca granítica, cartografiada por Rivas Martínez (1987) en la hoja correspondiente del Mapa de Series de Vegetación de España. 


\section{Quercetum ilicis galloprovinciale quercetosum pubescentis $\mathrm{Br}$ - $\mathrm{Bl}$.} 1936.

Bosque mixto de encinas y robles (Inventarios $\mathrm{n}^{\mathrm{o}}$ 1-13). En las zonas relativamente más elevadas y más secas el roble, aunque abundante, comparte con la encina la dominancia del estrato arbóreo. Las especies del Quercion ilicis son más frecuentes (Arbutus unedo, Asparagus acutifolius, Smilax aspera, Phillyrea angustifolia, Rubia peregrina, etc.). El mismo alcornoque puede estar presente en la asociación, si bien a nuestro parecer, habría sido introducido en la época de expansión de la industria del corcho en la provincia (s. XIX). Las especies de Querco-Fagetea, no obstante, son aún abundantes (Juniperus communis ssp. communis, Rubus ulmifolius, Ulmus minor, Crataegus monogyna, Euphorbia amygdaloides, Prunus spinosa, etc.) y actúan, junto con especies del matorral acidófilo del Lavandulo-Ericetum scopariae (Erica scoparia, Lavandula stoechas, Cytisus scoparius, etc.) como diferenciales.

En el dominio de este bosque son importantes las repoblaciones de Pinus pinaster e incluso de eucalipto.

\section{IS C US ION Y CONCLUS IONES}

La mayor parte del dominio climácico de estos bosques ha sido reconvertido a superficies de cultivo, por lo que sólo quedan pobladas con vegetación arbórea pequeñas áreas marginales o pequeñas superficies que se disponen en mosaicos entre los cultivos. Esta situación y la presión que ejerce la población agrícola, dificulta la obtención de buenos inventarios que reflejen un estado maduro de los bosques. En general, las formaciones forestales presentan una cubierta arbórea poco densa dominada por Pinus pinea (profusamente favorecido en la comarca) y $Q$. pubescens, de manera que el bosque aparece como un pinar mezclado con robles. El sotobosque se transforma en estos casos en un matorral del Lavandulo-Ericetum scopariae, el cual, por degradación, pasa generalmente a un prado anual de tréboles (Trifolio-Brachypodietum retusi) (Bolòs, 1959; Vilar, 1987).

Estos datos nos indican que algunas áreas mediterráneas más o menos extensas pueden albergar, en condiciones locales particulares de clima y suelo, una vegetación climácica dominada por Quercus caducifolios.

A G R A D E C I M I E N T O S . Agradecemos las orientaciones dadas por el Dr. J. Vigo para la realización de este trabajo.

\section{B I B L I O G R A F I A}

BOLOS, O. DE -1959- El paisatge vegetal de dues comarques naturals: La Selva i la Plana de Vic. Inst. Est. Cat., Arx. Ciènc., XXVI.

BOLOS, O. DE \& VIGO, J. -1984- Flora dels Països Catalans. 
TABLA DE LAS ASOCIACIONES Quercetum ilicis galloprovinciale quercetosum pubescent is $\left(n^{0} 1-13\right)$ y Carici-Quercetum canariensis holcetosum nova $\left(\mathrm{n}^{\mathrm{o}}{ }_{14-18)}\right.$.

Nứm. Orden

Altitud $(m)$
Orientación

Inclinación ${ }^{\circ}$

Estrato arboreo, altura (m)

Estrato arbóreo, cobertura $(\%)$

Estrato arbustivo, altura (m)

Estrato arbustivo, cobertura $(\%)$
Estrato herbáceo, cobertura $(\%)$

$\begin{array}{cccccccccccccccccc}1 & 2 & 3 & 4 & 5 & 6 & 7 & 8 & 9 & 10 & 11 & 12 & 13 & 14 & 15 & 16 & 17 & 18 \\ 135 & 130 & 130 & 130 & 120 & 110 & 75 & 70 & 130 & 90 & 100 & 90 & 95 & 125 & 125 & 120 & 120 & 100\end{array}$

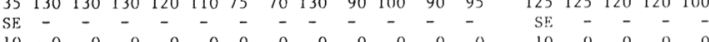

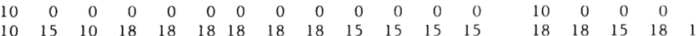

$\begin{array}{lllllllllllllllllll}70 & 70 & 60 & 80 & 70 & 70 & 60 & 80 & 70 & 60 & 70 & 90 & 90 & 60 & 90 & 95 & 95 & 100\end{array}$

$\begin{array}{llllllllllllllllll}2 & 2 & 2 & 3 & 3 & 2 & 2 & 2 & 2 & 2 & 2 & 2 & 1 & 3 & 1 & 2 & 2 & 2\end{array}$

Características del Quercion robori-petraeae y diferenciales respecto al Quercetea ilicis
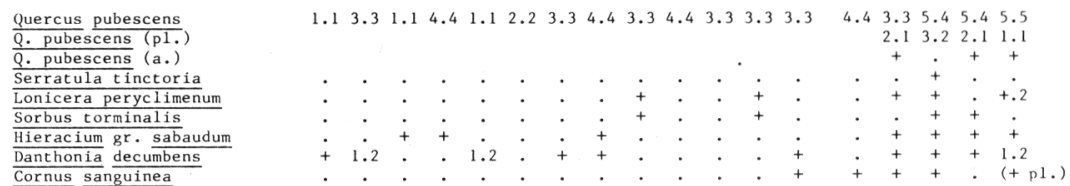

Dif. subas. holcetosum
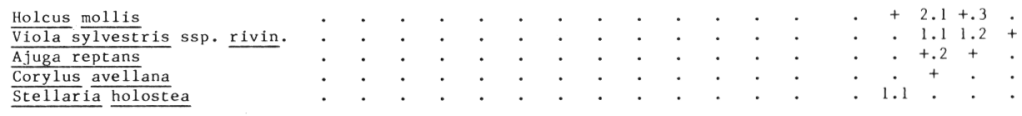

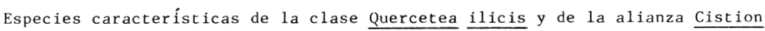
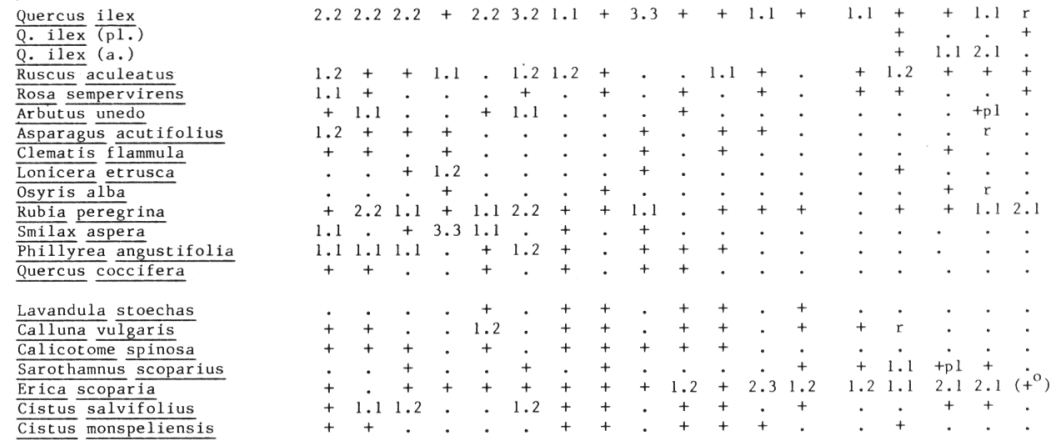

Compañeras

Lavandula stoechas

Calicotome spinosa

Sarothamnus scoparius

Erica scoparia

Cistus $\frac{\text { salvifolius }}{\text { monspeliensis }}$

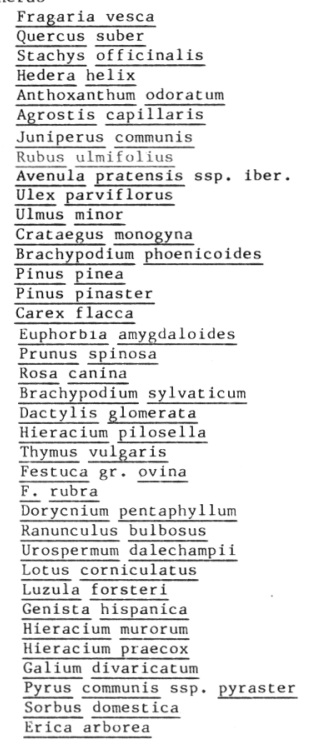

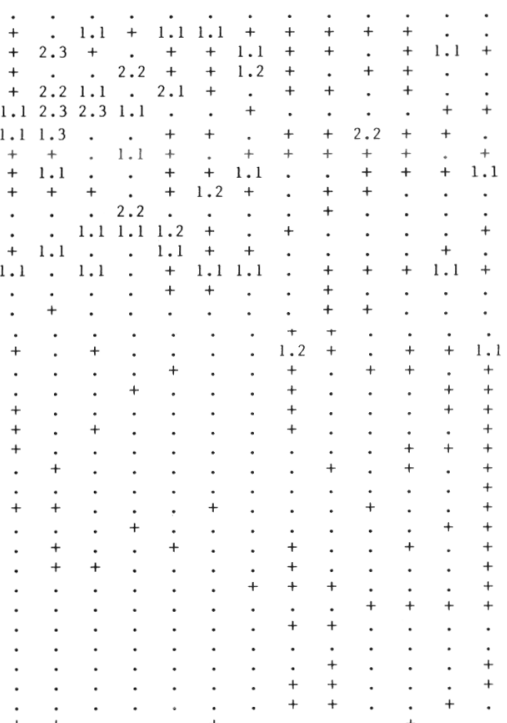

$\begin{array}{ccccc}+ & \mathbf{r} & + & \dot{r} & + \\ +1 & 1.1 & 1.1 & 2.1 & 1.1 \\ + & 3.3 & 2.3 & 4.4 & 3.3\end{array}$

$+\frac{+}{1.2} 1.2++$

$1.12 .2+1.13 .3$

Trica arborea

Presentes en uno o dos inventarios: Spartium junceum $(2,6)$, Galium maritimum (2), Mespilus germanica (4), Agrimonia

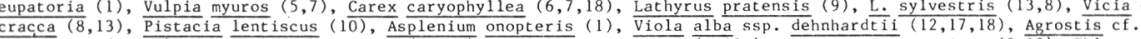
castellana $(12,15)$, Trisetaria flavescens $\left.(13,15), \frac{\text { Trifolium campestre }}{(8,13},\right)$, Chamaecytisus supinus $\left(9, \frac{\text { Che }}{13)}\right.$, Phleum nodosum $(1,13)$, Ononis spinosa (13), Lathyrus linifolius $(8,16)$, Fraxinus angustifolia $(8,18 \mathrm{pl})$, Koeleria splendens $(14)$

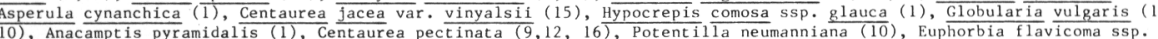
10), Anacamptis pyramidalis (1), Centaurea pectinata (9,12,16), Potentilla neumanniana (10), Euphorbia flavicoma ssp.

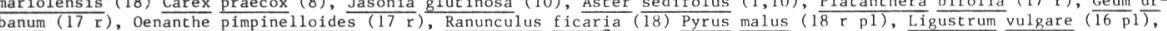

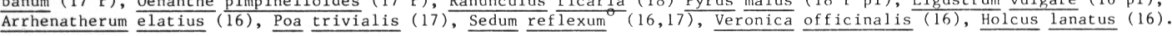

Procedencia de los inventarios (todos ellos en al província de Gerona, cuadrícula UTM 3IT DG):

1. Vilobí d'Onyar, Sta. Margarita. DG 73

2. Girona, Palau, junto al Colegio Montesori. DG 84

3. Girona, junto al cementerio de Palau Sacosta. DG 84

4. Cerca del anterior. DG 84

5. Girona, junto edificio de Trabajo, Palau. DG 84

6. Vilobí d'Onyar, junto al pueblo. DG 73

7. Riudellots de la Selva, junto a la carretera a Cassà de la Selva. DG 8

8. Caldes de Malavella, junto a la carretera a Llagostera, $\mathrm{Km} \mathrm{4,70,} \mathrm{DG} 83$

9. Riudarenes, cerca de L'Esparra. DG 73

. Con Sils. DG 83

12. Riudarenes, junto a la carretera a Sta. Coloma, Km. 7 . DG 73

13. Caldes de Malavella, junto urbanización "Llac del Cigne". DG 83

14. Riudellots de la Selva, Fuente del Mas Vilà. DG 83

15. Cerca del anterior. DG 83

16. Vilobí d'Onyar, junto a Can Tarré. DG 73

18. Caldes de Malavella, cerca de la carretera a Llagostera. DG 83 

BRAUN-BLANQUET, J., ROUSSINE, N. \& NEGRE, R. -1952- Les gruopements végétaux de la France méditerranéenne. C.N.R.S. Montpellier.

KIELHAUSER, G. -1939- Zur Okologie des Quercetum galloprovinciale pubescentetosum. S.I.G.M.A., com. 67. Oestern. Bot. Zeitsh. 88,1.

RIVAS-MARTINEZ, S. -1987- Memoria del mapa de series de vegetación de España. I.C.O.N.A. Min. Agri. Pesca y Alimen.

QUEZEL, P. -1985- Carte de la végétation potentielle de la région méditerranéenne. C.N.R.S. Paris.

VILAR, L. -1987- Flora i Vegetació de la Comarca de la Selva. Tesis Doctoral. Universidad Autónoma de Barcelona.

VILAR, L., VIÑAS, X., XERCAVINS, A., P0LO, L. -1988- Relació entre las Inversió Tèrmica i la Vegetació a la Plana de la Selva (Girona). Actes Simp. Intern. Bot. Pius Font i Quer. Lleida (en prensa).

ZELLER, W. -1959-Etude phytosociologique du Chêne-Liège en Catalogne. Librería General. Zaragoza.

(Aceptado para su publicación el 23 de noviembre de 1989)

Dirección de los autores: Lab. de Botànica, Estudi General de Girona (U.A.B.) Pl. Hospital, 6. 17071-Girona. 